\title{
The Severity of the Clinical Expression of Lysosomal Acid Lipase in Patients with Cryptogenic Cirrhosis
}

\author{
Arturo Solano-Urrusquieta ${ }^{1, ~ *, ~ N e l s o n ~ E d u a r d o ~ A l v a r e z-L i c o n a ~}{ }^{2}$, Jose Antonio Morales-Gonzalez ${ }^{3}$, \\ Raul Fierros-Oceguera ${ }^{4}$, Javier Bastida-Alquicira ${ }^{5}$, Alicia Sarahi Ojeda-Yuren ${ }^{6}$, Eira Cerda-Reyes ${ }^{7}$ \\ ${ }^{1}$ Internal Assistance Department, Secretary of National Defense, Mexico City, Mexico \\ ${ }^{2}$ Post-graduate Department, Higher School of Medicine, National Polytechnic Institute, Mexico City, Mexico \\ ${ }^{3}$ Laboratory of Conservation Medicine, Higher School of Medicine National Polytechnic Institute, México City, Mexico \\ ${ }^{4}$ Department of Palliative Care, "El Buen Samaritano" Hospital, Mexico City, Mexico \\ ${ }^{5}$ Radiology Department, Ultrasonography and non-Invasive Images Section, Central Military Hospital, City of Mexico, Mexico \\ ${ }^{6}$ Faculty of Bioethics, Anahuac University Mexico, Mexico City, Mexico \\ ${ }^{7}$ Gastroenterology Section, Central Military Hospital, Mexico City, Mexico
}

\section{Email address:}

doctorurrusquieta@gmail.com (A. Solano-Urrusquieta),nalvarez@ipn.mx (N. E. Alvarez-Licona), jmorales101@yahoo.com.mx (J. ^. Morales-Gonzalez), raulfierros5@hotmail.com (R. Fierros-Oceguera), obusero15@hotmail.com (J. Bastida-Alquicira),dra.asojeday@gmail.com (A. S. Ojeda-Yuren), arieirace@yahoo.com.mx (E. Cerda-Reyes) ${ }^{*}$ Corresponding author

\section{To cite this article:}

Arturo Solano-Urrusquieta, Nelson Eduardo Alvarez-Licona, Jose Antonio Morales-Gonzalez, Raul Fierros-Oceguera, Javier Bastida-Alquicira, Alicia Sarahi Ojeda-Yuren, Eira Cerda-Reyes. The Severity of the Clinical Expression of Lysosomal Acid Lipase in Patients with Cryptogenic Cirrhosis. American Journal of Internal Medicine. Vol. 9, No. 3, 2021, pp. 142-147. doi: 10.11648/j.ajim.20210903.16

Received: March 24, 2021; Accepted: April 21, 2021; Published: June 4, 2021

\begin{abstract}
Lysosomal acid lipase is an enzyme that intervenes in the last steps of lipid metabolism to hydrolyze cholesteryl esters. Patients with cryptogenic cirrhosis present a clear deficiency of lysosomal acid lipase with an unknown mechanism. Design: The present study has an analytical and retrospective design, of a sample of 55 patients with cryptogenic cirrhosis. The degree of association of lysosomal acid lipase was determined with the results of the enzymes alanine aminotransferase and alkaline phosphatase, as well as with the clinical manifestations of portal hypertension and splenic volume. The sensitivity and specificity of the test were determined for the diagnosis of the manifestations of portal hypertension. Results: The most frequent complication of portal hypertension was variceal hemorrhage with $40 \%$, followed by ascites with $32.7 \%$, and last, by hepatic encephalopathy, with $18.2 \%$. Association by the $\mathrm{x}^{2}$ test was without statistical significance with values of $0.177,0.299$, and 0.184 for encephalopathy, variceal hemorrhage, and ascites, respectively. The association of lysosomal acid lipase and splenic volume utilizing the Student $t$-test had a low degree of association and $p>0.05$. Through ROC curves, we obtained AUROC results close to 0.5 . Conclusion: It is established that there is no correlation of the levels of activity of lysosomal acid lipase with the values of alanine aminotransferase and alkaline phosphatase, as well as with the presence or absence of clinical manifestations and by ultrasound of portal hypertension. Lysosomal acid lipase is not a good test for the diagnosis of the clinical manifestations of portal hypertension.
\end{abstract}

Keywords: Lysosomal Acid Lipase, Cryptogenic Cirrhosis, Portal Hypertension, Liver Cirrhosis, Liver Cirrhosis

\section{Introduction}

Liver cirrhosis is considered a global public health problem with an increasing prevalence at a worldwide level, due to greater recognition of the diagnosis and the associated risk factors. Liver cirrhosis has an estimated prevalence in the North-American population of $0.27 \%$ [1] and a prevalence in the Latin population of chronic liver disease (CLD) of $61.1 \%$ [2]. CLD-associated mortality increased by $46 \%$ at the global level during the period comprising 1980-2010 and it is between the first 11 causes of death in 
some countries [3-4]. The increase in mortality is associated in part with the prevalence of non-alcoholic fatty Liver Disease (NAFLD), together with the pandemic of obesity and type 2 diabetes [5]. The main cause of liver cirrhosis in Mexico is alcoholic liver disease (ALD) and hepatitis $C$ virus (HCV) infection [6, 7]. Cryptogenic cirrhosis (CC) was the third most frequent cause in 2004 in Mexico [7], at present with non-alcoholic steatohepatitis (NASH) the third most frequent cause with $23.3 \%$ [8].

Lysosomal acid lipase (LAL) is the intralysosomal enzyme that intervenes in the final steps of lipid metabolism, and it is charged with hydrolyzing cholesteryl esters (CE) and triacylglycerides (TG) in free cholesterol and fatty acids, respectively [9-10]. It has been reported that LAL activity levels are found to be diminished in patients with some type of CLD, with an inverse relation with fibrosis [11] and the decrease of cell lineages of platelets and leukocytes [12], being frequently documented in NAFLD, NASH, hepatic fibrosis, liver cirrhosis, chronic $\mathrm{HCV}$ infection and in $\mathrm{CC}$ especially [11-15]. Mexican consensus on the diagnosis of lysosomal acid lipase deficiency (LALD) establishes the criteria for scrutinizing LAL in hepatopathies or alterations of liver functioning of undetermined etiology [16], and other authors recommend its screening, in addition to screening for type-IIa hypercholesterolemia-associated hepatopathies without established causes [17].

The most frequent findings in the physical exploration of patients with cholesteryl ester storage disease (CESD) are hepatomegaly (99.3\%) and splenomegaly (74\%) [18], and the most frequently defined extra-hepatic manifestations are complications of portal hypertension ( $\mathrm{PH})$, and among these, esophageal varices [18-19]. Also, the increase in alanine aminotransferase (ALT) and alkaline phosphatase (ALP) have been associated with CESD [18]. The greater prevalence of $\mathrm{PH}$ in patients with CLD and LALD has not been established by means of imaging studies [20]. LALD is not commonly suspected and has a low prevalence, which makes difficult its clinical study, particularly in those patients where their diseases have a low predictive value, according to the clinical context, and absence of family history $[14,19$, 21-22]. Lysosomal acid lipase deficiency presents a hypercholesterolemia phenotype similar to type IIa or type IIb, and low HDL, which are recognized as highly atherogenic diseases. Besides, these dyslipoproteinemia and hyperlipidemia are considered a risk factor for hepatocarcinoma and cholagiocarcinoma [23-26].

Due to the latter, the objectives of the present study were to establish the correlation of the diminution of the enzyme LAL with the enzymes of the alanine aminotransferase (ALT) and alkaline phosphatase (ALP) profiles, as well as with the clinical manifestations of $\mathrm{PH}$ and with ultrasonography findings, in order to increase the information related to $\mathrm{CC}$ and establish a physiopathological mechanism consequently.

\section{Materials and Methods}

The present study was carried out at the Central Military
Hospital of Mexico City, and the investigation protocol was approved by the Committee of Investigation with registration number C. INV.-072. The sample of the study was of 55 patients restricted to those who were over 15 years of age, of both sexes, who had been diagnosed with $\mathrm{CC}$. The results of the measuring of the variables were obtained during the same hospitalization period (2016 to 2018) during which the patients were diagnosed with CC. The patient's clinical files were obtained in order to review information on liver-disease categorization, biochemical data, and PH features. The patients who were discarded were those with comorbidities or complications (cardiac failure, hematological cancer, etc.) that were susceptible to modify the results of the variables and increase the biases; and those who had died during hospitalization due to other causes rather than $\mathrm{PH}$ findings.

\subsection{Primary and Secondary Endpoint}

The correlation was carried out of the activity levels of LAL with ALT and ALP. Similarly, the degrees were established of LAL with $\mathrm{PH}$, such as ascites, variceal bleeding, and hepatic encephalopathy, in addition to the correlation between LAL levels and the splenic ultrasound findings. Later, we estimated the sensitivity and specificity of the LAL test as the diagnostic value in the manifestations of $\mathrm{PH}$ with ROC curves.

\subsection{Statistical Analysis}

The results are represented as mean and the categorical variables, with an " $n$ " (\%). We determined the normality of the variable by means of the Kolmogorov-Smirnov test for LAL $(\mathrm{t}=0.145 ; \mathrm{p}>0.05)$, ALT $(\mathrm{t}=0.354 ; \mathrm{p}>0.05)$, ALP $(\mathrm{t}=0.173 ; \mathrm{p}>0.05)$, and splenic volume $(\mathrm{t}=0.147 ; \mathrm{p}>0.05)$.

The independent association was effected of the variables ALT, ALP, and splenic volume with LAL using the Pearson correlation and afterward by linear multiple regression analysis. To prove whether there was an association between the categorical variables of portal hypertension and the categorized LAL variable-under-study, we utilized the Fisher exact test. For the analytical correlation model between LAL and the clinical manifestations of $\mathrm{PH}$, we conducted binary logistic regression analysis. Later, we developed ROC curves for the test of LAL with the complications of PH.

\section{Results}

Table 1 presents the demographic and biochemical characteristics of the study's entry population, in which it is observed that 55 patients, as well as the main results of the correlations (women $n=48$, and men $n=7$ ), were distributed by sex and age with the following results (male: female ratio $1: 7$, and mean age $58.93 \pm 12.56$ years). We conducted the t-test of the mean of our variables of one sample with the mean of Vespasiani-Gentilucci, et al. [12], obtaining for LAL a $\mathrm{t}=-10.088 ; \mathrm{p}=0.005$. With this, it is established that the mean of LAL activity is significantly less. In the same manner, we performed the same analysis with splenic volume, 
obtaining a $\mathrm{t}=-6.300 ; \mathrm{p}=0.005$, which corresponds to that a splenic volume of the population of our work is significantly less than in the enzyme ALT, in which a $\mathrm{t}=2.113$; $\mathrm{p}=0.02$ was obtained, with which it is concluded that the mean of ALT is significantly greater than in our sample.

Table 1. Main characteristics of the study population and correlations.

\begin{tabular}{|c|c|c|c|}
\hline Variable $N=55$ & Mean & Correlation & $\mathbf{p}$ \\
\hline LAL* (nmol/spot/h) & $350.03 \pm 203.80$ & & \\
\hline Age (years) & $58.93 \pm 12.56$ & & \\
\hline $\mathrm{ALT}^{\beta}(\mathrm{IU} / \mathrm{L})$ & $90.07 \pm 168.32$ & 0.234 & $<0.05$ \\
\hline $\operatorname{ALP}^{\uparrow}(\mathrm{UI} / \mathrm{L})$ & $176.78 \pm 95.27$ & 0.079 & 0.282 \\
\hline Splenic volume $(\mathrm{mm})$ & $505.33 \pm 212.51$ & -0.139 & 0.394 \\
\hline Collateral circulation & $23(39.65 \%)$ & -1.42 & 0.738 \\
\hline INR $\dagger$ & $1.26 \pm 0.21$ & & \\
\hline BMIt & $28.9 \pm 4.15$ & & \\
\hline Hepatic encephalopathy & & $X^{2}=474$ & 0.093 \\
\hline Ascites & & $\chi^{2}=2.018$ & 0.365 \\
\hline Variceal bleeding & & $\chi^{2}=0.925$ & 0.63 \\
\hline
\end{tabular}

*LAL; Lysosomal Acid Lipase, ${ }^{\beta}$ ALT; Alanine aminotransferase, $\uparrow$ ALP; Alkaline phosphatase, $\dagger$ INR; International Normalized Ratio, $\ddagger$ BMI; Body Mass Index.

The proportion of patients classified according to the disease's severity is found in Figure 1. Of the 55 subjects, 26 belong to class A, 20 subjects to class B, and last, nine subjects belong to class $\mathrm{C}$ of the Child-Pugh scale. The enzyme LAL had a mean of $350.03 \pm 203.80$, ALT presented a mean of $90.07 \pm 168.32$, ALP had a mean of $176.78 \pm 95.27$, and splenic volume, a mean of $505.33 \pm 212.51$ (Figure 1).

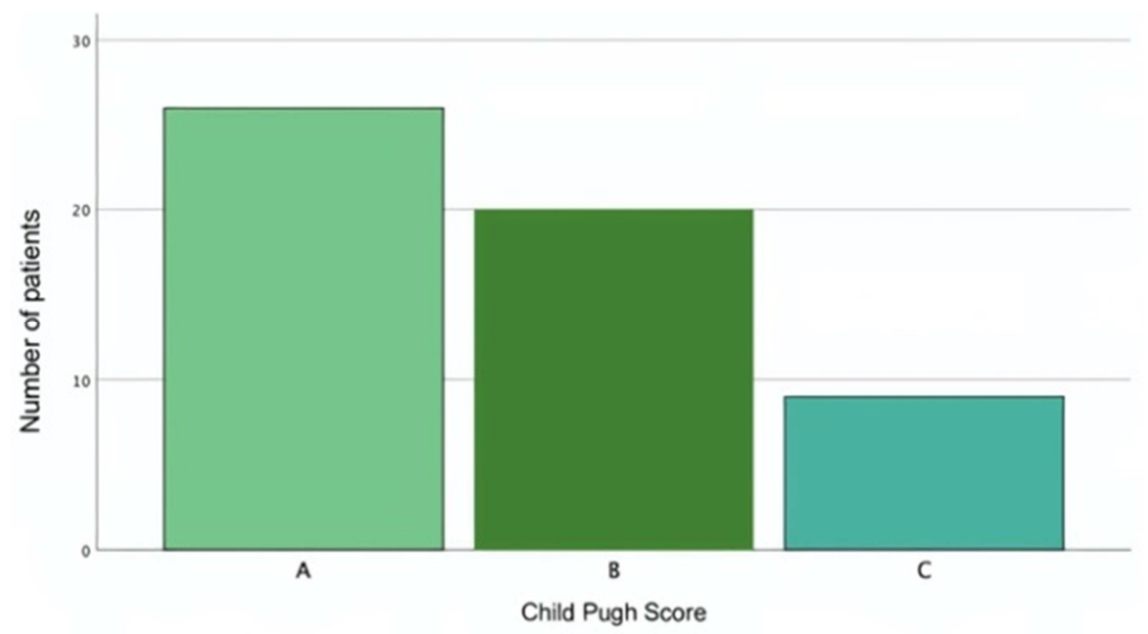

Figure 1. Bar graph representation of the proportions of severity by means of the Child-Pugh scale. Child-Pugh Score A 26 (47.06\%), Child-Pugh Score B $20(36.2 \%)$, and Child-Pugh Score C 9 (16.29\%).

The enzyme ALT does not present a clinically significant correlation with the variability of LAL $(95 \%$ CI $\mathrm{r}=0.234$; $\mathrm{p}<0.05)$ and, for its part, ALP does not present a clinical correlation nor statistical significance $(95 \%$ CI $\mathrm{r}=0.079$; $\mathrm{p}=0.282)$. The correlation of splenic volume with LAL resulted without statistical significance and with a low degree of correlation ( $r=-0.139 ; \mathrm{p}=0.394)$.

The proportion of complications associated with $\mathrm{PH}$ in the first place was variceal hemorrhage with $40 \%$, followed by ascites with $32.7 \%$, and last, hepatic encephalopathy with $18.2 \%$. A total of $65.5 \%$ of patients with esophageal varices were classified with enlarged varices of the Baveno classification. The linear multiple regression analysis of an $\mathrm{r}=0.395$, with an adjusted $\mathrm{R}$ of 0.31 (31\%), explains $31 \%$ of the variation of the manifestations of portal hypertension in turn explained by LAL variability.

The LAL variable was categorized with a cut-off point of $>500$ and of $<500 \mathrm{nmol} / \mathrm{spot} / \mathrm{h}$, obtaining one for hepatic encephalopathy of $100 \%$ and a specificity of $17.8 \%$, for variceal bleeding a sensitivity of $90.9 \%$, and a specificity of $18.2 \%$ and, last, a sensitivity of $94.4 \%$, and a specificity of $18.9 \%$. Patients with CC did not have a greater frequency of hepatic encephalopathy as the values diminished of LAL, the study LAL $(81.81 \%$ vs. $18.18 \% ; \mathrm{p}=0.177)$, similarly in patients with ascites $(67.3 \%$ vs. $32.7 \%$; $=0.184)$ and with variceal hemorrhage (59.99\% vs. $39.99 \%, \mathrm{p}=0.299)$.

The binary logistic model was not statistically significant with a $\chi^{2}=4.74 ; p=0.093$ for hepatic encephalopathy, $\chi^{2}=2.018$; $\mathrm{p}=0.365$ for ascites, and $\chi^{2}=0.925 ; \mathrm{p}=0.63$ for variceal bleeding. Afterward, employing ROC curves, we obtained the following results: for variceal hemorrhage, 0.517, hepatic encephalopathy, 0.333 , and for ascites, 0.429 , concluding that this is not a good diagnostic test for the clinical manifestations of portal hypertension (Figures 2-4). 


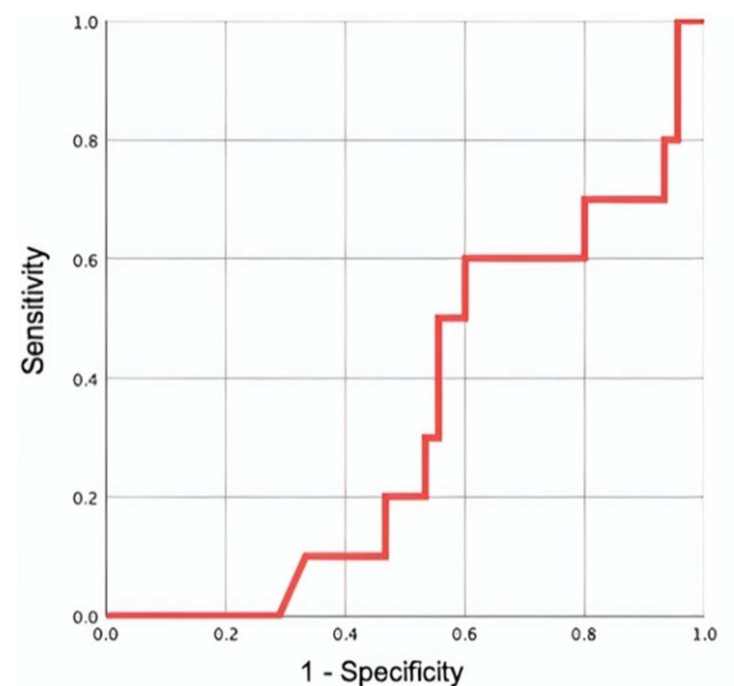

Figure 2. ROC curve for hepatic encephalopathy with a calculated area under the curve of 0.333 .

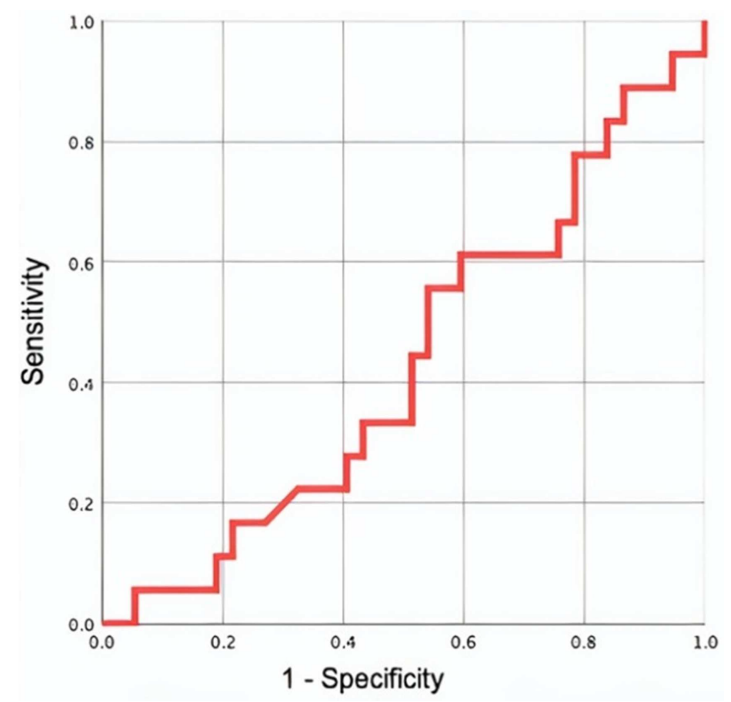

Figure 3. ROC curve for ascites with an area under the curve calculated at 0.429 .

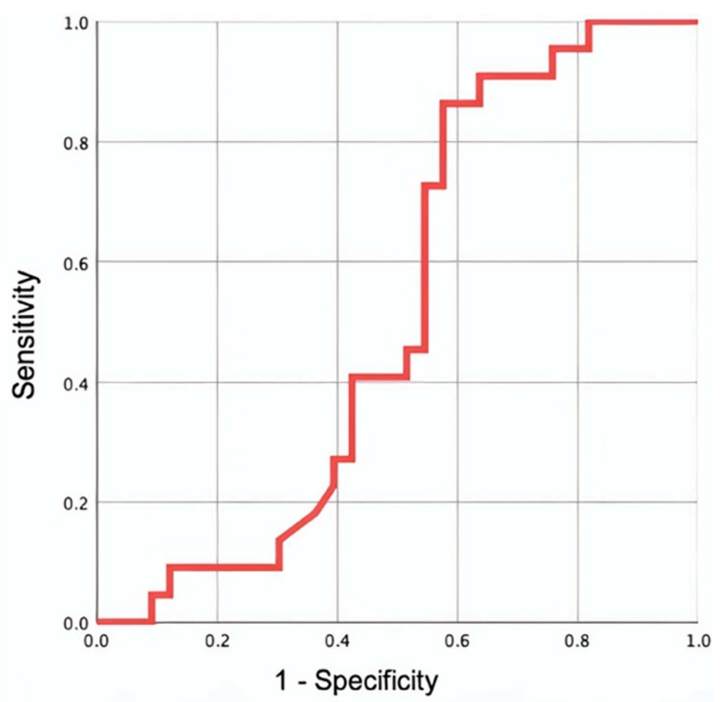

Figure 4. ROC curve with variceal bleeding with an area under the curve calculated at 0.517 .

\section{Discussion}

Liver cirrhosis is a public health problem at the worldwide level due to its high economic costs in diverse sectors, mainly in the health and labor sector $[4,7,27]$. In 2004, it was found to be the third cause of cirrhosis in Mexico and, as in the rest of the studies, women were those majorly affected $[4,11]$. In our study, the proportions of affectation by gender in patients with CC were maintained, because women constituted $87.3 \%$ $(n=48)$ of our total sample. In the study by Rinaldi, et al., the most frequent complications of $\mathrm{PH}$ in patients with $\mathrm{CC}$ were ascites with $54.9 \%$, followed by hepatic encephalopathy with $25.5 \%$, and last, variceal bleeding with $6.9 \%$ [28], in contrast with our population, in which variceal hemorrhage was the most frequent complication with $40 \%$, followed by ascites with $32.7 \%$ and hepatic encephalopathy with $18.2 \%$. The importance of the latter lies in that variceal hemorrhage is the complication associated with greater mortality; our patients have been documented with esophageal varices, classifying enlarged varices according to the classification of Baveno. The lipid-limiting enzyme LAL is found diminished in multiple chronic hepatic disorders [11], and CC is the hepatic affectation with the greatest diminution of LAL compared with other etiologies [11, 12]. Although the results of Vespasiani-Gentilucci, et al. describe that, according to the diminution of the enzyme LAL, patients present data of lesser severity. Nonetheless, the state of severity is not well-defined through the severity scale [12]. Mean LAL in patients with NAFLD was $530 \mathrm{nmol} / \mathrm{spot} / \mathrm{h}$ [19], and mean LAL in CC according to our results was $350.03 \mathrm{nmol} / \mathrm{spot} / \mathrm{h}$. Similarly, in the statistical analysis, the mean LAL in our population is significantly less in comparison with the mean of the sample of Vespasiani-Gentilucci, et al [12]. On the other hand, the enzyme ALT is significantly greater than the mean of those same authors, and the splenic volume of our population is lower; therefore, these changes could be the cause of obtaining different results and probably due to that, in Mexico, there is a greater number of patients with obesity, metabolic syndrome, and diabetes than in the population of Italian origin [12]. Patients with $\mathrm{CC}$ are frequently diagnosed at earlier disease stages than those with $\mathrm{CH}$ due to other etiologies [28], this is congruent with our results because our team demonstrated that 46 subjects in the sample were found in Child-Pugh class A-B. According to the study published by Bernstein, et al. patients with LALD are associated with a rise in ALT and AST [18], while in our results, mean ALT and ALP were found increased: the diminution of the LAL enzyme in patients with CC had no correlation with the increase of ALT and ALP, concluding that the diminution of LAL does not entail the measurement of hepatic enzymes. With the latter, the result was not able to be validated as compared with that of Bernstein, et al CC is a diagnosis of exclusion that, in a not-at-all negligible percentage, is due to NAFLD [18]; likewise, the enzyme LAL, while very much below the mean of other works on Mexican population, appears to have no relation with the variability of the hepatic profile, manifestations of portal hypertension, and splenic volume. 
Vespasiani-Gentilucci, et al. described that the diminution of LAL is associated with the greater presence of esophageal varices [12]. However, our study demonstrated the same, with $74.6 \%(n=41)$ of patients presenting esophageal varices at the time of diagnosis. Whether enlarged or small varices according to the classification of Baveno, the relation of LAL was sought out intentionally with variceal hemorrhage. A null statistical correlation was established between the diminution of LAL and a greater amount of patients with variceal bleeding. Similar to Shteyer, et al., in our work we were unable to demonstrate data on portal hypertension by means of the imaging studies of patients with reduced LAL activity levels due to an increase in splenic volume or data of collateral circulation by splenic ultrasound [20]. The results of Polimeni, et al. proved the diminution of LAL, even in patients without cirrhosis or $\mathrm{PH}$ [15]; therefore, the physiopathological mechanism for the diminution of LAL is multifactorial and is not liver- and/or leukocyte-dependent. Although it is well-known that the principal cause of $\mathrm{PH}$ is cirrhosis, patients with NAFLD initiate with an increase in splenic volume from early disease stages, even presenting without $\mathrm{PH}$ or $\mathrm{CH}$. LAL entertains an inverse association with the splenic volume [15]. According to our results, on determining the $\mathrm{R}$ factor at the moment of diagnosis, it was found that $92.73 \%(n=51)$ of patients presented an $R$ factor of $<2$, compatible with possible damage of cholestatic origin, with hepatocellular the least frequent. In addition, while this result does not demonstrate a physiopathological mechanism, its analysis could be considered using other methods in order to validate results. According to an analysis of the clinical and biochemical associations in patients with LALD or those with cirrhosis of any etiology, it could be expected that LAL would exercise cholestatic damage on the liver, according to the findings of the relation with the increase of splenic volume, of esophageal varices, and of variceal bleeding in these, in addition to an increase in $\gamma$-glutamyl transpeptidase. However, there are many confounder mechanisms, such as the fact that the patients with $\mathrm{CH}$ can present leukopenia and thrombocytopenia due to splenomegaly, independently of the cause of the cirrhosis, which can condition a greater diminution, as in cases caused by HCV. Likewise, LAL activity has been established in these cell lines (leukocytes and platelets), being greater in the leukocytes [29]; thus, leukopenia could be a confounding factor.

\section{Conclusions}

It is established that there was no statistical significance between the correlation of LAL and the enzymes alanine aminotransferase and the alkaline phosphatase, as well as the complications of PH. The enzyme LAL is not a good test for the diagnosis of the clinical manifestations of $\mathrm{PH}$.

In our population, the most frequent complication of portal hypertension was variceal bleeding, in contrast with other works in patients with cryptogenic cirrhosis. Therefore, it would be important to recognize which are the risk factors that increase the rate of variceal hemorrhage in our population, because this complication is considered as that of greatest mortality in patients with liver cirrhosis. Scarce investigation in patients with cryptogenic cirrhosis, since it is a diagnosis of exclusion and given that it could easily be a manifestation of another pathology without being able to arrive at the etiological diagnosis, makes the studies entertain broad variability in their results; thus, it would be difficult to validate the publication.

\section{Conflicts of Interest}

There are no conflicts of interest.

\section{References}

[1] G. Eason, B. Noble, and I. N. Sneddon, "On certain integrals of Lips Scaglione S, Kliethermes S, Cao G, Shoham D, Durazo R, Luke A et al. The Epidemiology of Cirrhosis in the United States. Journal of Clinical Gastroenterology. 2015; 49 (8): 690-696.

[2] Setiawan V, Stram D, Porcel J, Lu S, Le Marchand L, Noureddin M. Prevalence of chronic liver disease and cirrhosis by underlying cause in understudied ethnic groups: The multiethnic cohort. Hepatology. 2016; 64 (6): 1969-1977.

[3] Marcellin P, Kutala BK. Liver diseases: a major, neglected global public health problem requiring urgent actions and large-scale screening. Liver Int 2018; 38 (Suppl 1): 2-6. https://doi.org/10.1111/liv.13682.

[4] Gao E, Hercun J, Heller T, Vilarinho S. Undiagnosed liver diseases. Transl Gastroenterol Hepatol [Internet]. 2021 Apr 1 [cited 2021 Apr 16]; 6 (28): 1-14. Available from: /pmc/articles/PMC7829073/.

[5] Kim D, Li AA, Gadiparthi C, Khan MA, Cholankeril G, Glenn JS, et al. Changing trends in etiology-based annual mortality from chronic liver disease, from 2007 through 2016. Gastroenterology https://doi.org/10.1053/j.gastro.2018.07.008.

[6] Cholankeril G, Wong RJ, Hu M, Perumpail RB, Yoo ER, Puri P, Younossi ZM, Harrison SA, Ahmed A. Liver Transplantation for Nonalcoholic Steatohepatitis in the US: Temporal Trends and Outcomes. Dig Dis Sci 2017; 62: 29152922 [PMID: 28744836 DOI: $10.1007 / \mathrm{s} 106200174684 x]$.

[7] Mendez-Sánchez N, et al. Etiology of Liver Cirrhosis in Mexico. 2004; Annals of Hepatology January (3): 30-33.

[8] Méndez-Sánchez N, Zamarripa-Dorsey F, Panduro A, Purón-González E, Coronado-Alejandro EU, Cortez-Hernández CA, et al. Current trends of liver cirrhosis in Mexico: Similitudes and differences with other world regions. World J Clin Cases [Internet]. 2018 Dec 1 [cited 2021 Apr 17]; 6 (15): 922-30. Available from: https://pubmed.ncbi.nlm.nih.gov/30568947/.

[9] Camarena C, Aldamiz-Echevarria L, Polo B, Barba Romero M, García I, Cebolla $\mathrm{J}$ et al. Update on lysosomal acid lipase deficiency: diagnosis, treatment and follow-up of patients. Clinical Medicine. 2017; 148 (9: 429. e1-429. e10.

[10] Ponziani F, Pecere S, Gasbarrini A, Ojetti V. Physiology and pathophysiology of liver lipid metabolism. Expert Review of Gastroenterology \& Hepatology. 2015; 9 (8): 1055-1067. 
[11] Angelico F, Corradini S, Pastori D, Fargion S, Fracanzani A, Angelico $\mathrm{M}$ et al. Severe reduction of blood lysosomal acid lipase activity in cryptogenic cirrhosis: A nationwide multicenter cohort study. Atherosclerosis. 2017; 262: 179-184.

[12] Vespasiani-Gentilucci U, Gallo P, Piemonte F, Riva E, Porcari A, Vorini F et al. Lysosomal Acid Lipase Activity Is Reduced Both in Cryptogenic Cirrhosis and in Cirrhosis of Known Etiology. PLOS ONE. 2016; 11 (5): e0156113.

[13] Bernstein DL, Lobritto S, Iuga A, Remotti H, Schiano T, Fiel MI, et al. Lysosomal acid lipase deficiency allograft recurrence and liver failure- clinical outcomes of 18 liver transplantation patients. Mol Genet Metab [Internet]. 2018; 124 (1): 11-9. Available from: https://doi.org/10.1016/j.ymgme.2018.03.010.

[14] Baratta F, Pastori D, Del Ben M, Polimeni L, Labbadia G, Di Santo $\mathrm{S}$ et al. Reduced Lysosomal Acid Lipase Activity in Adult Patients with Non- alcoholic Fatty Liver Disease. EBioMedicine. 2015; 2 (7): 750-754.

[15] Polimeni L, Pastori D, Baratta F, Tozzi G, Novo M, Vicinanza $\mathrm{R}$ et al. Spleen dimensions are inversely associated with lysosomal acid lipase activity in patients with non-alcoholic fatty liver disease. Internal and Emergency Medicine. 2017; 12 (8): 1159-1165.

[16] Vázquez-Frias $\mathrm{R}$, García-Ortiz J, Valencia-Mayoral $\mathrm{P}$, Castro-Narro G, Medina-Bravo P, Santillán-Hernández Y et al. Mexican consensus on the diagnosis of lysosomal acid lipase deficiency. Journal of Gastroenterology of Mexico. 2018; 83 (1): 51-61.

[17] Baratta F, Pastori D, Polimeni L, Tozzi G, Violi F, Angelico F et al. Does Lysosomal Acid Lipase Reduction Play a Role in Adult Non-Alcoholic Fatty Liver Disease? International Journal of Molecular Sciences. 2015; 16 (12): 28014-28021.

[18] Bernstein D, Hulkova H, Bialer M, Desnick R. Cholesteryl ester storage disease: Review of the findings in 135 reported patients with an underdiagnosed disease. Journal of Hepatology. 2013; 58 (6): 1230-1243.

[19] Tovoli F, Napoli L, Negrini G, D’Addato S, Tozzi G, D’Amico $\mathrm{J}$, et al. A relative deficiency of lysosomal acid lypase activity characterizes non-alcoholic fatty liver disease. Int J Mol Sci [Internet]. 2017 Jun 1 [cited 2021 Apr 15]; 18 (6): 1-15. Available from: /pmc/articles/PMC5485958/.

[20] Shteyer E, Villenchik R, Mahamid M, Nator N, Safadi R. Low Serum Lysosomal Acid Lipase Activity Correlates with Advanced Liver Disease. International Journal of Molecular Sciences. 2016; 17 (3): 312.
[21] Strebinger G, Müller E, Feldman A, Aigner E. $<p>$ Lysosomal acid lipase deficiency - early diagnosis is the key $</ \mathrm{p}>$. Hepatic Med Evid Res [Internet]. 2019 May [cited 2021 Apr 19]; Volume 11: 79-88. Available from: /pmc/articles/PMC6536894/.

[22] Pastores GM, Hughes DA. Lysosomal acid lipase deficiency: Therapeutic options [Internet]. Vol. 14, Drug Design, Development and Therapy. Dove Medical Press Ltd.; 2020 [cited 2021 Apr 19]. p. 591-601. Available from: /pmc/articles/PMC7023879/.

[23] Schulz P, Ferreira F, Nascimento M, Vieira A, Ribeiro M, David A et al. Association of nonalcoholic fatty liver disease and liver cancer. World Journal of Gastroenterology. 2015; 21 (3): 913

[24] Pericleous M, Kelly C, Wang T, Livingstone C, Ala A. Wolman's disease and cholesteryl ester storage disorder: the phenotypic spectrum of lysosomal acid lipase deficiency. Lancet Gastroenterol Hepatol [Internet]. 2017; 2 (9): 670-9. Available from: http://dx.doi.org/10.1016/S2468-1253(17)30052-3.

[25] Perumpail BJ, Khan MA, Yoo ER, Cholankeril G, Kim D, Ahmed A. Clinical epidemiology and disease burden of nonalcoholic fatty liver disease [Internet]. Vol. 23, World Journal of Gastroenterology. Baishideng Publishing Group Co; 2017 [cited 2021 Apr 19]. p. 8263-76. Available from: /pme/articles/PMC5743497/.

[26] Liu Z, Alsaggaf R, Mcglynn KA, Anderson LA, Tsai HT, Zhu $\mathrm{B}$, et al. Statin use and reduced risk of biliary tract cancers in the UK Clinical Practice Research Datalink. Gut [Internet]. 2019 Aug 1 [cited 2021 Apr 18]; 68 (8): 1458-64. Available from: /pmc/articles/PMC6525087/.

[27] Smith A, Baumgartner K, Bositis C. Cirrhosis: Diagnosis and Management. Am Fam Physician. 2019; 100 (12): 759-70.

[28] Rinaldi L, Nascimbeni F, Giordano M, Masetti C, Guerrera B, Amelia A et al. Clinical features and natural history of cryptogenic cirrhosis compared to hepatitis $\mathrm{C}$ virus-related cirrhosis. World Journal of Gastroenterology. 2017; 23 (8): 1458 .

[29] Vespasiani-Gentilucci U, D'Amico J, De Vincentis A, Tozzi G, Vorini F, Gallo P et al. Platelet count may impact on lysosomal acid lipase activity determination in dried blood spot. Clinical Biochemistry. 2017; 50 (12): 726-728. 